\title{
Operative Technique for Single Incision Robot-Assisted Transaxillary Thyroid Surgery
}

\author{
${ }^{1}$ Christine S Landry, ${ }^{1}$ David S Kwon, ${ }^{1}$ Elizabeth G Grubbs, ${ }^{2}$ G Stephen Morris \\ ${ }^{1}$ Jeffrey E Lee, ${ }^{1}$ Nancy D Perrier \\ ${ }^{1}$ Department of Surgical Oncology, Section of Surgical Endocrinology, The University of Texas MD Anderson Cancer Center, Texas, USA \\ ${ }^{2}$ Department of Physical Therapy, The University of Texas MD Anderson Cancer Center, Texas, USA
}

Correspondence: Nancy D Perrier, Department of Surgical Oncology, Section of Surgical Endocrinology, The University of Texas MD Anderson Cancer Center, 1515 Holcombe Boulevard, Unit 444, Houston, Texas-77030, USA, Phone: 713-794-1345 Fax: 713-563-5727, e-mail: nperrier@mdanderson.org

\section{ABSTRACT}

During the past five years, transaxillary approaches to thyroid surgery have been introduced into surgical literature. These techniques were initially performed using traditional endoscopy, and most recently with a surgical robot. This manuscript describes our approach to robotassisted transaxillary surgery (RATS) for thyroidectomy using a single axillary incision. Because of the steep learning curve, this procedure is best implemented with a team approach. The ideal team consists of a console surgeon who operates the robot, a bedside surgeon who assists with retraction and troubleshoots robotic arm collisions and a circulating assistant who helps optimize the efficiency of the operation.

Keywords: Minimally invasive thyroidectomy, Transaxillary, Operative techniques, Robotic thyroidectomy.

\section{INTRODUCTION}

The evolution of thyroid surgery represents an outstanding example of medical progress through the synergistic combination of art, science, technology and healing. Prior to the development of adequate hemostatic and antiseptic techniques, open thyroidectomy carried an extremely high risk of morbidity and death. ${ }^{1}$ However, contributions from surgeons, such as Billroth, Kocher, Halsted and Mayo rapidly transformed thyroidectomy into a safe operation. ${ }^{1}$ In fact, the standard technique of open thyroidectomy changed only modestly from the standard open approach that was established by these surgical pioneers over the next century. The introduction of video-assisted and endoscopic cervical approaches to thyroidectomy in the new millennium has changed the operative technique employed by many surgeons in order to minimize cervical scars. ${ }^{1,2}$

During the past 5 years, transaxillary approaches to thyroid surgery have been described in the surgical literature. ${ }^{3}$ These techniques were initially performed using traditional endoscopy, and more recently with a surgical robot. ${ }^{3}$ Robot-assisted transaxillary surgery (RATS) for removal of the thyroid gland can achieve superior cosmetic results in patients by avoiding a cervical scar. In addition, RATS may reduce postoperative neck discomfort and swallowing symptoms when compared to the open approach. ${ }^{4}$ On the other hand, RATS requires a longer operative time, is associated with a greater expense and patients may have temporary shoulder discomfort and chest paresthesia compared to the traditional open procedure. ${ }^{4}$

RATS offers several advantages to the surgeon. It provides excellent three-dimensional visualization of the operative field as well as optimal precision due to enhanced instrument mobility provided by the robotic wrists. Owing to the ergonomic design of the robotic console, surgeons may experience less fatigue. The primary drawback of RATS from the standpoint of the thyroid surgeon is the requirement of specialized equipment, the need for significant training, the steep learning curve and the necessity for specialty team expertise.

This manuscript describes our approach to RATS using a single axillary incision. Because of the steep learning curve, this procedure is best implemented with a team approach. The ideal team consists of a console surgeon who operates the robot, a bedside surgeon who assists with retraction and troubleshoots robotic arm collisions, and a circulating assistant who helps to optimize the efficiency of the operation.

\section{ROBOTIC TRAINING}

Prior to the implementation of RATS into clinical practice, every surgeon on the team must undergo proper training. After becoming familiar with the relevant literature, each surgeon should become proficient with the surgical system by training in the robotics lab. Every member of the team should then gain additional familiarity with the procedure by observing several operations performed by an experienced surgeon. Further experience should be acquired by performing RATS on cadavers. Prior to performing the first procedure in a patient, the operating room staff should be trained in the use of the robotic equipment and in set-up for the technique itself. The first few cases should be proctored by an experienced surgeon. We introduced RATS into our surgical practice by initially performing all operations as a team, with each member assigned 
a specific role (Table 1). The roles were rotated for each case so that all surgeons would master every part of the procedure. This team-based approach was advantageous to enhance learning of each portion of the operation and to help maximize efficiency. After completing 12 to 15 cases as a team, we became proficient in RATS. We now comfortably perform RATS as single surgeons with the assistance of a trained co-surgeon, such as a fellow, resident or partner-staff surgeon.

\section{PREOPERATIVE PLANNING AND SET-UP}

\section{Patient Selection}

Many factors should be considered when selecting patients for RATS. For instance, a patient's body habitus significantly impacts the ease of operative dissection. Thin individuals with a body mass index less than 30 and a small body frame are ideal candidates. Also, the operation becomes exponentially more difficult in patients whose distance from the sternal notch to the lateral edge of the pectoralis major muscle exceeds $18 \mathrm{~cm}$. Likewise, RATS is best performed in patients who have a maximum thyroid lobe diameter of $5 \mathrm{~cm}$.

Patients who have large goiters that extend posterior to the trachea are not candidates for RATS due to the difficulty of the dissection and posterior exposure. Also, patients with preexisting shoulder problems should not undergo RATS due to the risk of exacerbating the pre-existing injury during the operation from patient positioning. Even though Kang et al have demonstrated the feasibility of RATS for thyroid cancer, ${ }^{3}$ we recommend performing the initial RATS cases for thyroid lesions of low malignant potential. Also, all women should be up-to-date on their mammograms prior to the operation because the operative dissection associated with RATS may temporarily affect the interpretation of these studies.

\section{Instrumentation}

To perform RATS, we use the da Vinci robotic system (Intuitive Surgical, Sunnyvale, CA), which has received FDA approval. The device has a central console and three robotic arms.
Table 2 lists the additional equipment required to perform RATS. During the initial dissection, several different retractors (insulated Army-Navy, Biggs, malleable, sweetheart, Deaver, and Richardson) are helpful when creating the flap. During the medial portion of the dissection, a cautery extender, long forceps, laparoscopic clip appliers and Harmonic scalpel (Ethicon, Cincinnati, $\mathrm{OH}$ ) are beneficial. A bladed thyroid retractor (Kuppersmith, Marena Medical, Florida) is required to expose the thyroid gland via the axillary incision and to allow for insertion of the robotic arms.

The equipment required for the robotic portion of the operation includes one $5 \mathrm{~mm}$ cannula to hold the $5 \mathrm{~mm}$ Maryland dissector, one $8 \mathrm{~mm}$ cannula to clutch the Harmonic scalpel, one $8 \mathrm{~mm}$ cannula to house the $8 \mathrm{~mm}$ ProGrasp ${ }^{\mathrm{TM}}$ forceps (Intuitive Surgical) and one disposable $12 \mathrm{~mm}$ trocar to secure a camera. The bedside surgeon also needs a laparoscopic grasper, irrigation/suction device and Endoscopic Kittners ${ }^{\mathrm{TM}}$ or bolsters (kites; approximately $1 \mathrm{~cm}$ long and $3 \mathrm{~mm}$ wide).

\section{Incision Placement}

Before the patient is taken to the operating suite, the incision site should be marked while the patient is awake and sitting upright with the arms hanging at his or her sides. The incision should be concealed on the ipsilateral side of the thyroid lesion in the region of the lateral-most border of the pectoralis major muscle. Placing the incision near the axillary hairline promotes cosmesis. Marking the patient while in an upright position allows for the pendulous breast and the fold of the arm to assume a normal position, which enhances concealment of the incision.

\section{Anesthetic Considerations}

A dedicated anesthesia team with proven camaraderie with the surgical team is ideal. Intravenous access should be obtained in the arm on the contralateral side of the planned thyroid lobectomy. The arm on the ipsilateral side of the planned thyroid lobectomy should be comfortably positioned and secured adjacent to the head with the patient awake. Improper

Table 1: Surgeon's roles in RATS for thyroidectomy

\begin{tabular}{|c|c|c|}
\hline Team member & Role & Location \\
\hline Console surgeon & $\begin{array}{l}\text { - Assists with open dissection } \\
\text { - Performs robotic dissection }\end{array}$ & $\begin{array}{l}\text { - Scrubbed during open dissection } \\
\text { - Console for robotic portion }\end{array}$ \\
\hline Bedside surgeon & $\begin{array}{l}\text { - Performs open dissection } \\
\text { - Positions and troubleshoots robotic arm positioning } \\
\text { - Assists console surgeon during robotic portion by } \\
\text { laryngeal nerve, carotid artery and internal jugular vein } \\
\text { - Maintains a clean operative field during the robotic portion } \\
\text { - Closes the wound }\end{array}$ & $\begin{array}{l}\text { - Scrubbed during entire case at bedside } \\
\text { on ipsilateral side of table }\end{array}$ \\
\hline Circulating surgeon & $\begin{array}{l}\text { - Retracts during open dissection to achieve exposure } \\
\text { - Deploys bladed thyroid retractor } \\
\text { - } \text { Drives robot into position on operative field } \\
\text { - } \text { positioning } \\
\text { - Assists bedside surgeon }\end{array}$ & $\begin{array}{l}\text { - Scrubbed for open dissection } \\
\text { - Unscrubbed for robot docking } \\
\text { - Scrubbed during robotic dissection and } \\
\text { closing }\end{array}$ \\
\hline
\end{tabular}


Table 2: Instruments required to perform RATS

\begin{tabular}{ll}
\hline Equipment for open dissection & \\
\hline Retractors & $\begin{array}{l}\text { Army-Navy (insulated) } \\
\text { Right-angled lighted retractors } \\
\text { Malleable }\end{array}$ \\
& Sweetheart (Harrington) \\
& Deaver \\
& Richardson \\
& Biggs \\
& Forceps (long) \\
Operative instruments & Bovie extender \\
& Metal-tipped suction device (long) \\
& Extended nerve monitor tip \\
& Laparoscopic 5 mm clip applier \\
& Zero degree camera \\
& Bladed thyroid retractor with suction tubing \\
\hline Equipment for robotic portion & \\
\hline Laparoscopic suction device & \\
Laparoscopic clip applier & \\
Laparoscopic grasper & \\
12 mm disposable trocar & \\
$30^{\circ}$ robotic camera & \\
8 mm robotic harmonic scalpel & \\
5 mm robotic ProGrasp & \\
3 robotic cannulas & \\
Endoscopic Kittner/bolster & \\
\hline
\end{tabular}

positioning of the ipsilateral arm may result in a brachial plexus injury. Once the patient confirms comfortable positioning of the arm, the arm is secured and the patient is intubated. A nerve monitoring endotracheal tube is optional.

\section{Patient Positioning}

To optimize the height of the bladed thyroid retractor, the $\mathrm{X}$-ray cassettes under the operative table may need to be removed prior to transferring the patient to the table. The necessity of removing these cassettes should be determined prior to the patient entering the operating room. The patient is positioned on the lateral edge of the table ipsilateral to the side of the planned axillary incision. A wedged back support is placed under the scapula, and the patient's head is slightly hyperextended to elevate the thyroid anteriorly. The neck should remain in a neutral position. The arm contralateral to the side of the axillary incision is padded and tucked. If the breasts are pendulous, the ipsilateral breast can be secured with tape for inferior retraction.

The patient's ipsilateral arm is positioned so that the shoulder is flexed to approximately $160^{\circ}$, internally rotated and slightly abducted (Fig. 1A). The elbow is flexed to approximately $90^{\circ}$, the upper arm is positioned at an angle to the head, and the forearm is placed immediately superior to the head. Arm positioning is done with the patient awake so that the patient can confirm that the position is comfortable and there is no evidence of brachial plexus hyperextension. The arm is then secured and immobilized on an arm board and padded at pressure points. Meticulous attention to detail is imperative to avoid neurologic injury. Optimizing arm position minimizes the operative dissection by establishing the shortest distance from the ipsilateral axilla to the anterior neck.

\section{Anatomic Landmarks}

Once body positioning is established and the patient is asleep, the anatomic landmarks of the proposed dissection should be marked with a pen (Fig. 1B). The marks delineate the planes of dissection from the lateral border of the pectoralis major muscle to the sternal notch inferomedially and to the thyroid cartilage superiorly.

\section{Other Considerations}

Communication between the console surgeon and the bedside surgeon is essential for a successful operation. The surgeons should request silence in the operating room. Also, a sign on the operating room door to limit access to nonessential personnel is critical to maintain concentration for the OR staff. Before anesthesia is induced, the patient's name, procedure and laterality should be confirmed.

\section{OPERATIVE PROCEDURE}

\section{Initial Dissection}

With the aid of a headlight, the bedside surgeon, who is seated on a stool, performs the initial dissection. After the 5 to $6 \mathrm{~cm}$ axillary incision is made, the bedside surgeon dissects through the subcutaneous tissue and fat to expose the lateral border of the pectoralis major muscle. Flaps are created immediately superficial to the pectoralis fascia along the proposed trapezoidal planes of dissection. Army-Navy, Biggs, right-angle 

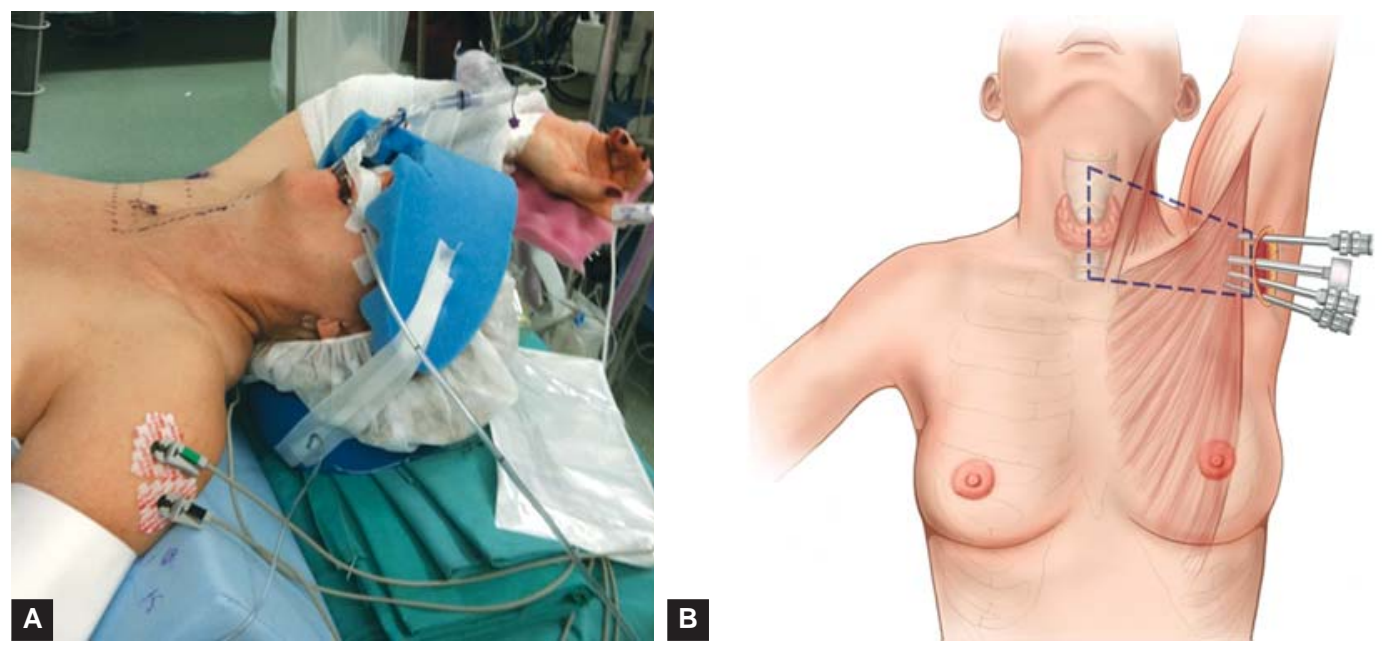

Figs 1A and B: Operative preparation: (A) Patient positioning. Proper arm positioning is a key component to success. The arm should not be hyperextended and all pressure points should be padded. (B) Planes of dissection. The planes of dissection are marked prior to the start of the operation

lighted and Deaver retractors are useful for medial retraction of the flap. Once the clavicle and sternum are identified, the sternal and clavicular heads of the sternocleidomastoid muscle are exposed. Care must be taken to avoid penetrating the skin over the clavicle while creating the flaps, especially in thin patients. The avascular plane between the sternal and clavicular heads of the sternocleidomastoid muscle is separated carefully, and the dissection is continued cranially (Fig. 2). The belly of the omohyoid muscle is retracted superiorly; occasionally, it is divided to achieve adequate exposure.

Once the lateral border of strap muscles is exposed, the strap muscles are elevated off of the thyroid parenchyma to expose the ipsilateral thyroid lobe. The dissection is continued medially until the medial third of the contralateral thyroid lobe is visualized. After the superior and inferior poles of the thyroid

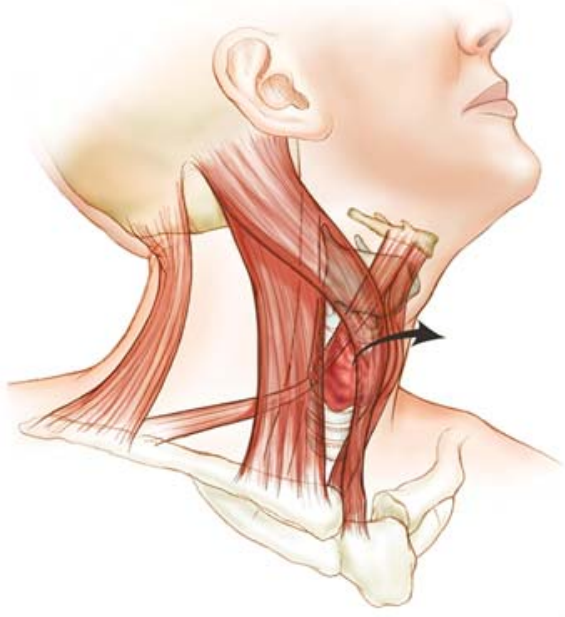

Fig. 2: Separating the heads of the sternocleidomastoid muscle. The avascular plane between the sternal and clavicular heads of the sternocleidomastoid muscle is separated carefully, and the dissection is continued cranially. The belly of the omohyoid muscle is retracted superiorly; occasionally, it is divided to achieve adequate exposure. Once the lateral border of strap muscles is exposed, the strap muscles are elevated off of the thyroid parenchyma to expose the ipsilateral thyroid lobe gland are exposed, a bladed thyroid retractor is deployed below the strap muscles to create a working space for the robotic arms. Achieving enough elevation to provide adequate working space is important for exposure.

\section{Thyroid Retractor}

The thyroid retractor is secured to the contralateral rail of the bed perpendicular to the thyroid isthmus (Fig. 3A). The blade is inserted into the operative field and positioned to elevate the strap musculature. If the blade is not secured at a $90^{\circ}$ angle to the thyroid, optimal positioning of the robotic arms may be hindered. Readjustment of the retractor is often necessary until the thyroid lobe is exposed for the robotic portion of the procedure.

The blade of the retractor is positioned under the sternal head of the SCM and the strap muscles, and elevated to create an operative space. A distance of several centimeters should be evident between the thyroid parenchyma and the strap muscles (Fig. 3B).

\section{Robot Docking}

The robot is positioned just short of the operating table contralateral to the operative side. The center console of the robot must be aligned with the thyroid gland and the bladed thyroid retractor. Robotic arm 3 must be locked into place on the posterior column. Next, the robotic arms are separated to achieve maximal range of motion while keeping the marked numbers on each arm facing forward. The center console must be positioned to accommodate the central tower area within the blue zone or "sweet spot", which allows maximal range of motion of the robotic arms.

\section{Insertion, Alignment and Deployment of Instruments}

A disposable $12 \mathrm{~mm}$ trocar (suction valve aimed up), two $8 \mathrm{~mm}$ cannulas and one $5 \mathrm{~mm}$ cannula are secured into the 
robotic arms as depicted in Figure 3. Next, a $30^{\circ}$ down camera is inserted into the camera arm. The Maryland dissector is placed into the $5 \mathrm{~mm}$ cannula, and the Harmonic scalpel and ProGrasp forceps are secured into the $8 \mathrm{~mm}$ cannulas.

Proper alignment of the robotic arms is absolutely essential for a successful operation. The operative space for the robotic arms is limited, and improper angles of insertion may lead to collision and damage of instruments. The role of the circulating surgeon is important during this portion of the case to ensure that the robotic arms are positioned correctly.

The camera is first angled $20^{\circ}$ down at the most lateral part of the incision in line with the bladed thyroid retractor. The angles and locations of the other instruments vary with the operative side (Figs 4A and B). Once each robotic arm is positioned to maximize mobility, instruments are deployed into the operative field under direct visualization. The robotic arms should be in precise, well-defined positions to allow for maximal articulation. Arm 3 is always on the opposite side of the thyroid retractor from the patient's head. Arms 2 and 1 are at the bottom corners of the semicircular space created.

\section{Console Surgeon}

The console surgeon performs the robot-assisted portion of the operation in the same way as a conventional open thyroidectomy, but using robotic instruments. First, the ProGrasp ${ }^{\mathrm{TM}}$ retractor is used to grasp the thyroid and retract it medially to expose the undersurface of the gland. The parathyroid glands are carefully dissected laterally using the Maryland dissector and Harmonic scalpel while preserving their vascular pedicle. The recurrent laryngeal nerve is identified along its course. The superior pole of the thyroid is carefully dissected to isolate the superior thyroidal vessels and to identify the superior laryngeal nerve. Each branch of the superior thyroid artery is individually ligated with the Harmonic scalpel. The inferior thyroid pole is separated from its inferior attachments, and the trachea is exposed. Since the surgeon has no tactile sensation when using the robot, continuous observation of the tracheal rings is necessary. If needed, the bedside surgeon can use a peanut device to palpate the tracheal rings for anatomic confirmation. The thyroid lobe is then dissected off the trachea while taking care not to injure the recurrent laryngeal nerve.

RATS is more difficult on the right side because the positioning of the robotic arms limits the console surgeon to two left-sided robotic instruments rather than the two right-sided instruments used on the left side. Occasionally, the location of instruments may need to be exchanged between the robotic arms to achieve better angles of dissection.

\section{Bedside Surgeon}

The bedside surgeon is responsible for maintaining a clear operative field with laparoscopic suction. The bedside surgeon also acts to increase exposure for the console surgeon by retracting the carotid artery laterally using a laparoscopic bolster. The bolster is also helpful in providing the countertraction needed during dissection along the recurrent laryngeal nerve. In addition, the bolster may be used to retract the internal jugular vein laterally to avoid injury. An extended suction tip is used to facilitate retraction by moving the sternocleidomastoid muscle out of the field. The bedside surgeon also uses laparoscopic graspers to remove the thyroid lobe after it is separated from the trachea.

After the thyroid lobe is removed, the bedside surgeon may use a laparoscopic irrigation device to irrigate and assure hemostasis. The function of the recurrent laryngeal nerve may

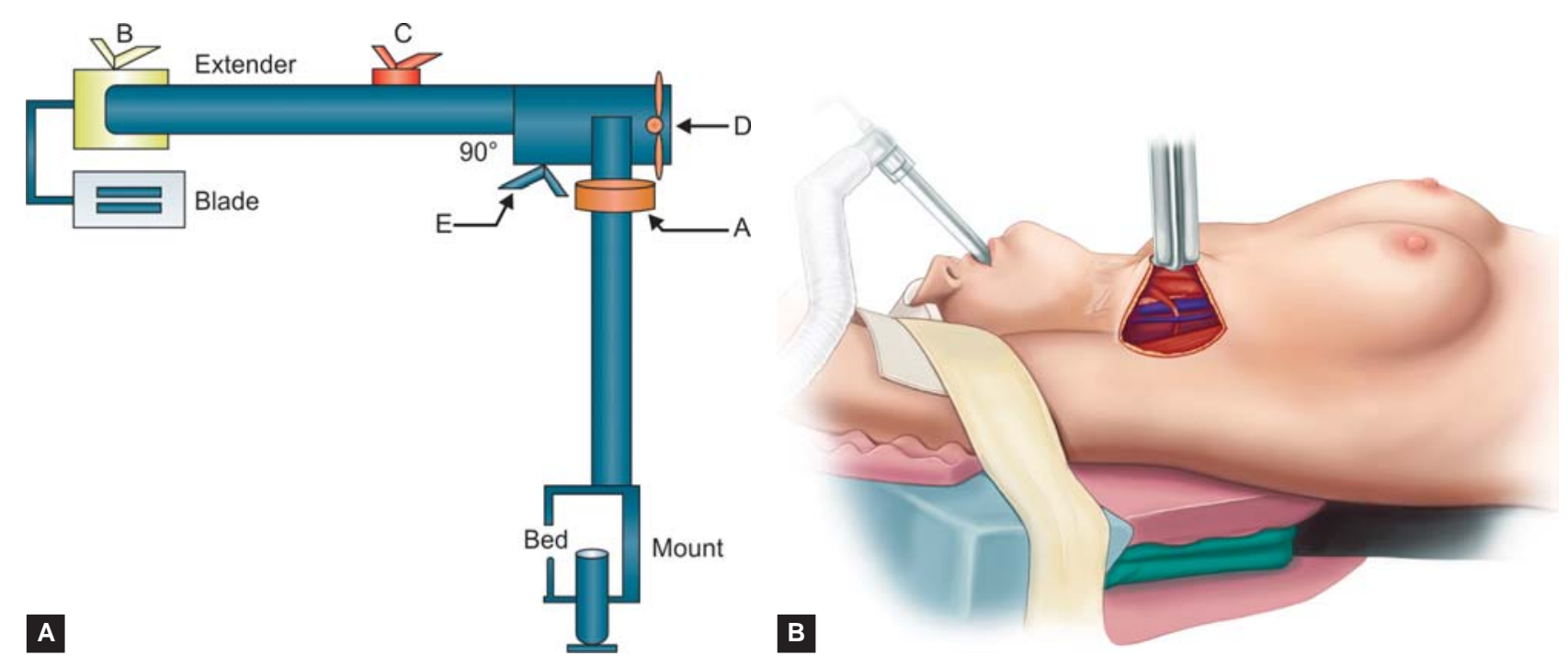

Figs 3 A and B: Thyroid retractor apparatus: (A) Operation of the thyroid bladed retractor-(1) attach mount on the retractor pole to the bed rail contralateral to the operative side (must align with center console and thyroid gland); (2) raise the bolt to the appropriate height and position in the axilla; (3) tighten the screw located at the blade; (4) tighten the screw located on the extender; (5) confirm that a $90^{\circ}$ angle is achieved between the retractor pole and extender. Ensure that the blade is positioned under the strap musculature to achieve the best exposure; (6) tighten the screw at to elevate the retractor in the body; (7) attach suction device to the retractor; (8) repeat the above steps as necessary to achieve optimal exposure. (B) The thyroid retractor in place. Adequate exposure of the supraclavicular space is necessary for visualization and for instrumentation 


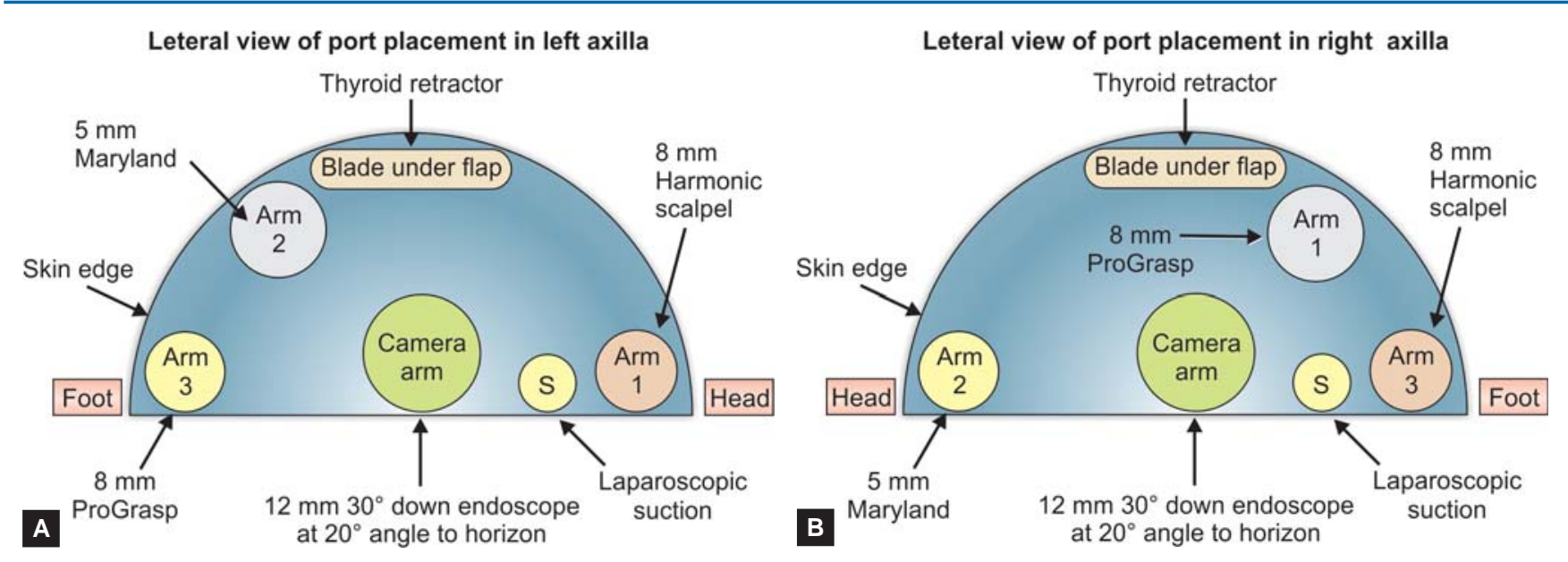

Figs 4A and B: Postioning of robotic arms. The positioning of the instruments is important to prevent collisions and to provide maximum mobility. The ProGrasp ${ }^{\top M}$ should be away from the head of the patient, relative to the thyroid reractor: (A) Positioning of robotic arms, left side. This diagram illustrates the postioning of each robotic arm relative to each other into the operative field. (B) Positioning of robotic arms, right side

be confirmed using a nerve monitor if desired. The circulating assistant can facilitate preparation of the nerve monitor for use, and assist with removal of the robotic arms.

\section{Closing}

Local anesthetic may be injected along the planes of dissection to assist with postoperative pain. A 10 French, round JacksonPratt drain is positioned in the thyroid bed and brought out laterally to the axillary incision. The bedside surgeon then closes the incision in layers with absorbable sutures. We have found that a protective hydrocolloid dressing is helpful during the postoperative period, especially for women whose incision is prone to irritation from a brassiere.

\section{POSTOPERATIVE CONSIDERATIONS}

Patients are kept in the hospital overnight and discharged the following day if pain is controlled. The drain is removed when output is minimal. Removing the drain too early may result in a seroma. Minor bleeding during the immediate postoperative period is generally not a concern because the blood will drain away from the neck under the flap and can be evacuated later if necessary. We recommend that women wear a sports brassiere during the first week postoperatively to protect the incision.

\section{ACKNOWLEDGMENTS}

We would like to thank Linda McGraw, for her administrative assistance, and Gary Hickok, for technical support.

\section{REFERENCES}

1. Hegner CF. A history of thyroid surgery. Ann Surg April 1932;95(4):481-92.

2. Miccoli P, Berti P, Raffaelli M, Conte M, Materazzi G, Galleri D. Minimally invasive video-assisted thyroidectomy. Am J Surg June 2001;181(6):567-70.

3. Kang SW, Jeong JJ, Yun JS, et al. Robot-assisted endoscopic surgery for thyroid cancer: Experience with the first 100 patients. Surg Endosc Nov 2009;23(11):2399-406.

4. Lee J, Nah KY, Kim RM, Ahn YH, Soh EY, Chung WY. Differences in postoperative outcomes, function, and cosmesis: Open versus robotic thyroidectomy. Surg Endosc 19 May 2010 [Epub ahead of print]. 